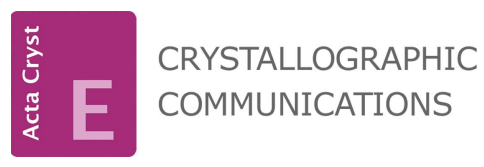

ISSN 2056-9890

Received 15 August 2020

Accepted 2 September 2020

Edited by L. Van Meervelt, Katholieke Universiteit Leuven, Belgium

Keywords: chloridostannate(IV); 1 -(prop-2-en-1yl)-1H-imidazole; Hirshfeld surface; fingerprint plots; optical absorption.; crystal structure.

CCDC reference: 2021940

Supporting information: this article has supporting information at journals.iucr.org/e

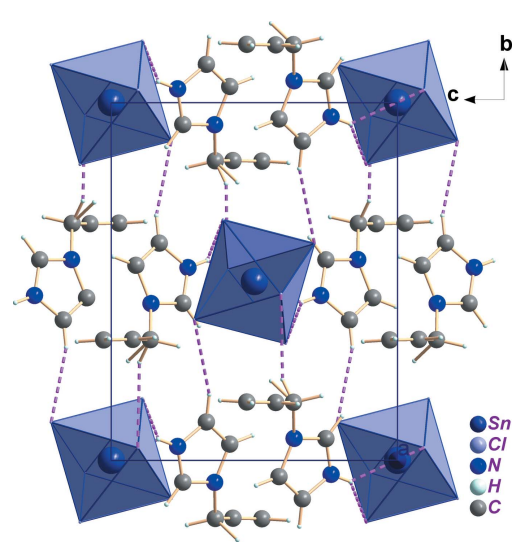

OPEN $\partial$ ACCESS

\section{Crystal structure, optical property and Hirshfeld surface analysis of bis[1-(prop-2-en-1-yl)-1H- imidazol-3-ium] hexachloridostannate(IV)}

\author{
Hela Ferjani*
}

Chemistry Department, College of Science, IMSIU (Imam Mohammad Ibn Saud Islamic University), Riyadh 11623, Kingdom of Saudi Arabia. *Correspondence e-mail: hhferjani@imamu.edu.sa

A new 0D organic-inorganic hybrid material bis[1-(prop-2-en-1-yl)-1Himidazol-3-ium] hexachloridostannate(IV), $\left(\mathrm{C}_{6} \mathrm{H}_{9} \mathrm{~N}_{2}\right)_{2}\left[\mathrm{SnCl}_{6}\right]$, has been prepared and characterized by single-crystal X-ray diffraction, Hirshfeld surface analysis and UV-visible spectroscopy. The structure consists of isolated $\left[\mathrm{SnCl}_{6}\right]^{2-}$ octahedral anions separated by layers of organic 1-(prop-2-en-1-yl)$1 H$-imidazol-3-ium cations. The 1-(prop-2-en-1-yl) fragment in the organic cation exhibits disorder over two sets of atomic sites having occupancies of 0.512 (9) and 0.488 (9). The crystal packing of the title compound is established by intermolecular $\mathrm{N} / \mathrm{C}-\mathrm{H} \cdots \mathrm{Cl}$ hydrogen bond and $\pi-\pi$ stacking interactions. Hirshfeld surface analysis employing three-dimensional molecular surface contours and two-dimensional fingerprint plots has been used to analyse the intermolecular interactions present in the structure. The optical properties of the crystal were studied using UV-visible absorption spectroscopy, showing one intense band at $208 \mathrm{~nm}$, which is attributed to $\pi-\pi^{*}$ transitions in the cations.

\section{Chemical context}

Tin(IV) halide organic-inorganic hybrid compounds are significant materials because of their interesting structural topologies and their wide range of optical applications such as luminescence, non-linear activity and semiconductivity (Hajji et al., 2016, 2019; BelhajSalah et al., 2018). As part of a continuing search of new organic-inorganic hybrid compounds such as $\mathrm{Bi}_{2} \mathrm{Cl}_{10}{ }^{4-}$ (Ferjani \& Boughzala, 2018; Ferjani et al., 2020) and $\mathrm{Hg}_{2} \mathrm{Cl}_{6}$ (Garci et al., 2019), the synthesis and characterization of a new hybrid material, bis[1-(prop-2-en-1-yl)-1H-imidazol-3-ium] hexachloridostannate(IV), $\left(\mathrm{C}_{6} \mathrm{H}_{9} \mathrm{~N}_{2}\right)_{2}\left[\mathrm{SnCl}_{6}\right]$ is reported.

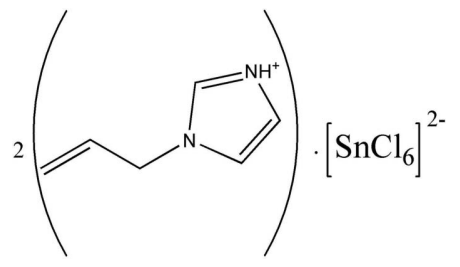

Imidazole was chosen as the organic cation because the resulting complexes show interesting structural, chemical and physical properties significant for photoluminescence, magnetism, ferroelectricity, and conductivity (Tritt-Goc et al., 2019; Babar et al., 2019; Ishak et al., 2019). The Hirshfeld surface analysis was performed to completely characterize the intermolecular interactions and explain the crystalline architecture. Moreover, the UV-visible spectrum was also investigated. 
$b \int_{a}^{c}$

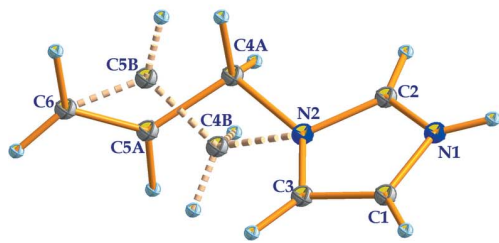

Figure 1

The asymmetric unit of $\left(\mathrm{C}_{6} \mathrm{H}_{9} \mathrm{~N}_{2}\right)_{2}\left[\mathrm{SnCl}_{6}\right]$, showing the atom-labelling scheme and the disorder of the allyl group with occupancies of 0.512 (9) (solid bonds) and 0.488 (9) (broken bonds).

\section{Structural commentary}

The asymmetric unit of $\left(\mathrm{C}_{6} \mathrm{H}_{9} \mathrm{~N}_{2}\right)_{2}\left[\mathrm{SnCl}_{6}\right]$ contains one $\left(\mathrm{C}_{6} \mathrm{H}_{9} \mathrm{~N}_{2}\right)^{+}$cation and one half of an $\left[\mathrm{SnCl}_{6}\right]^{2-}$ anion (Fig. 1). The $\mathrm{Sn}^{\mathrm{IV}}$ atom is located on a special position of site symmetry $2{ }_{1} / n$ (crystallographic center of inversion) and is coordinated by six chlorine atoms in an octahedral geometry. The hexachlorostannate(IV) octahedron is nearly perfect with $\mathrm{Sn}-\mathrm{Cl}$ bond lengths ranging from 2.4136 (6) to 2.4363 (6) $\AA$ and $\mathrm{Cl}-$ $\mathrm{Sn}-\mathrm{Cl}$ bond angles between 88.44 (3) and $180^{\circ}$. These bond lengths and angles are in good agreement with those observed in similar compounds based on hexachlorostannate(IV) (van Megen et al., 2013; Zhou et al., 2012; Rademeyer et al., 2007). The organic $\left(\mathrm{C}_{6} \mathrm{H}_{9} \mathrm{~N}_{2}\right)^{+}$cations are related to each other by $2_{1} /$ $n$ symmetry elements. The overall negative charges in the structure are counter-balanced by the protonated 1-(prop-2en-1-yl)-1H-imidazol-3-ium cations (Fig. 1). As usual, this aromatic amine is protonated at the $\mathrm{N} 1$ atom. The $\mathrm{C}=\mathrm{C}$ and

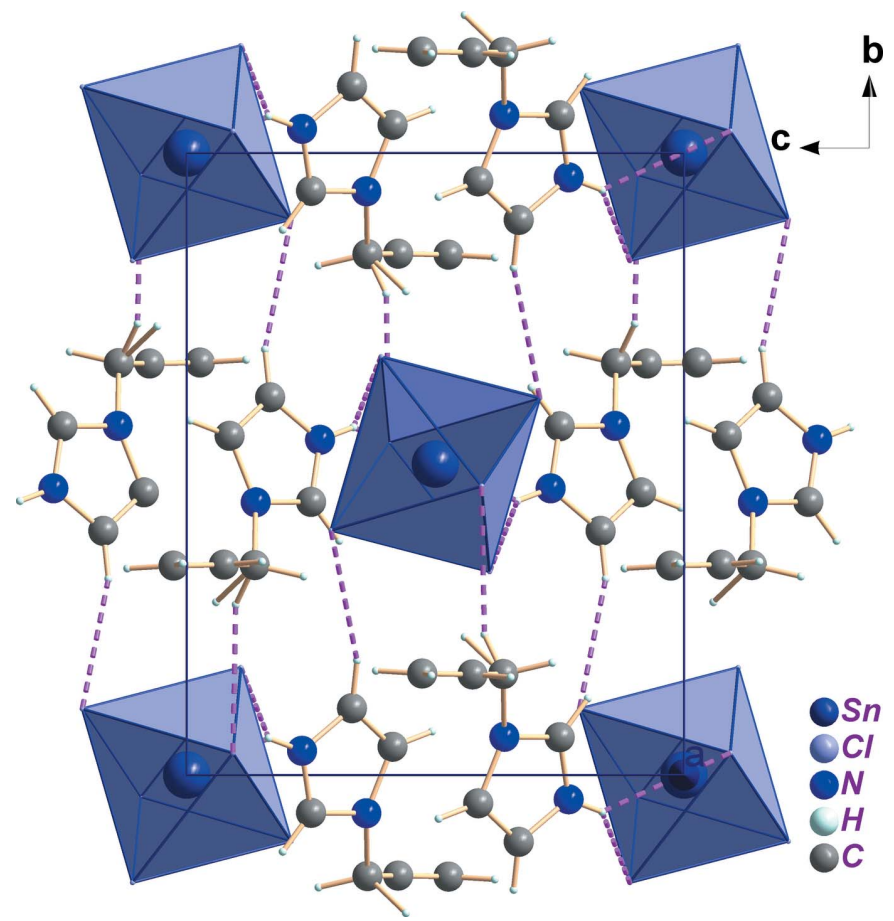

Figure 2

Crystal packing of $\left(\mathrm{C}_{6} \mathrm{H}_{9} \mathrm{~N}_{2}\right)_{2}\left[\mathrm{SnCl}_{6}\right]$ viewed along the $a$ axis, showing the $\mathrm{N}-\mathrm{H} \cdots \mathrm{Cl}$ and $\mathrm{C}-\mathrm{H} \cdots \mathrm{Cl}$ hydrogen bonds (dashed lines).
Table 1

Hydrogen-bond geometry $\left(\AA{ }^{\circ}\right)$.

\begin{tabular}{lllll}
\hline$D-\mathrm{H} \cdots A$ & $D-\mathrm{H}$ & $\mathrm{H} \cdots A$ & $D \cdots A$ & $D-\mathrm{H} \cdots A$ \\
\hline $\mathrm{N} 1-\mathrm{H} 1 A \cdots \mathrm{Cl} 2$ & 0.86 & 2.67 & $3.399(4)$ & 144 \\
$\mathrm{~N} 1-\mathrm{H} 1 A \cdots \mathrm{Cl} 1$ & 0.86 & 2.81 & $3.485(4)$ & 136 \\
$\mathrm{C} 2-\mathrm{H} 2 \cdots \mathrm{Cl}^{\mathrm{i}}$ & 0.93 & 2.79 & $3.672(4)$ & 158 \\
$\mathrm{C} 3-\mathrm{H} 3 \cdots 3^{\mathrm{ii}}$ & 0.93 & 2.90 & $3.790(4)$ & 160 \\
$\mathrm{C} 4 A-\mathrm{H} 4 A 1 \cdots \mathrm{Cl} 2^{\mathrm{iii}}$ & 0.97 & 2.89 & $3.716(9)$ & 144 \\
$\mathrm{C} 4 A-\mathrm{H} 4 A 1 \cdots \mathrm{Cl}^{\mathrm{iv}}$ & 0.97 & 2.94 & $3.743(10)$ & 141 \\
$\mathrm{C} 4 B-\mathrm{H} 4 B 1 \cdots \mathbf{C l}^{\mathrm{v}}$ & 0.97 & 2.98 & $3.702(10)$ & 133 \\
$\mathrm{C} 4 B-\mathrm{H} 4 B 2 \cdots \mathrm{Cl}^{\mathrm{iv}}$ & 0.97 & 2.80 & $3.590(7)$ & 139 \\
$\mathrm{C} 5 B-\mathrm{H} 5 B \cdots \mathrm{Cl}^{\text {vi }}$ & 0.93 & 2.97 & $3.766(11)$ & 145 \\
\hline
\end{tabular}

Symmetry codes: (i) $x-\frac{1}{2},-y+\frac{1}{2}, z-\frac{1}{2}$; (ii) $x-1, y, z-1$; (iii) $-x+\frac{3}{2}, y+\frac{1}{2},-z+\frac{1}{2}$; (iv) $x-\frac{1}{2},-y+\frac{3}{2}, z-\frac{1}{2}$; (v) $-x+1,-y+1,-z+1$; (vi) $-x+1,-y+1,-z$

ring $\mathrm{C}-\mathrm{N}$ bond lengths vary from 1.253 (8) to 1.307 (5), and 1.265 (6) to 1.349 (5) $\AA$, respectively, which agree well with those in homologous materials involving 1-(prop-2-en-1-yl)$1 H$-imidazole (Ferjani, 2020; Parshina et al., 2019). The crystal structure can be described as an organization of organicinorganic layers, which propagate along the $a$ axis at $y=0$ and $y=1 / 2$ (Fig. 2). These layers are formed by $\left[\mathrm{SnCl}_{6}\right]^{2-}$ octahedra and $\left(\mathrm{C}_{6} \mathrm{H}_{9} \mathrm{~N}_{2}\right)^{+}$organic cations.

\section{Supramolecular features}

The cohesion and stabilization of the title structure is ensured by $\mathrm{N}-\mathrm{H} \cdots \mathrm{Cl}$ and $\mathrm{C}-\mathrm{H} \cdots \mathrm{Cl}$ hydrogen bonds between the $\mathrm{NH}^{+}$unit of $1 \mathrm{H}$-imidazol-3-ium as the donor group and the chlorine atoms of the $\left[\mathrm{SnCl}_{6}\right]^{2-}$ octahedron as acceptor with $\mathrm{H}$. . Cl lengths ranging between 2.67 and $2.98 \AA$ (Fig. 2 and

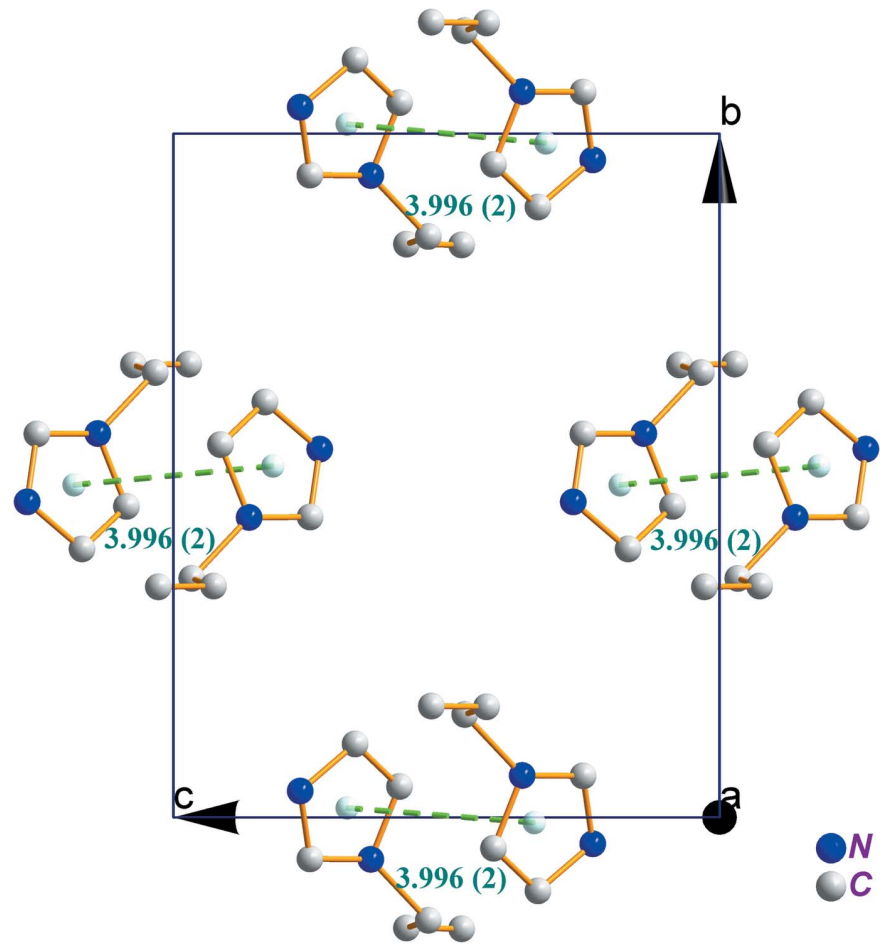

Figure 3

The $\pi-\pi$ interactions between organic cations in $\left(\mathrm{C}_{6} \mathrm{H}_{9} \mathrm{~N}_{2}\right)_{2}\left[\mathrm{SnCl}_{6}\right] . \mathrm{H}$ atoms are omitted for clarity. 

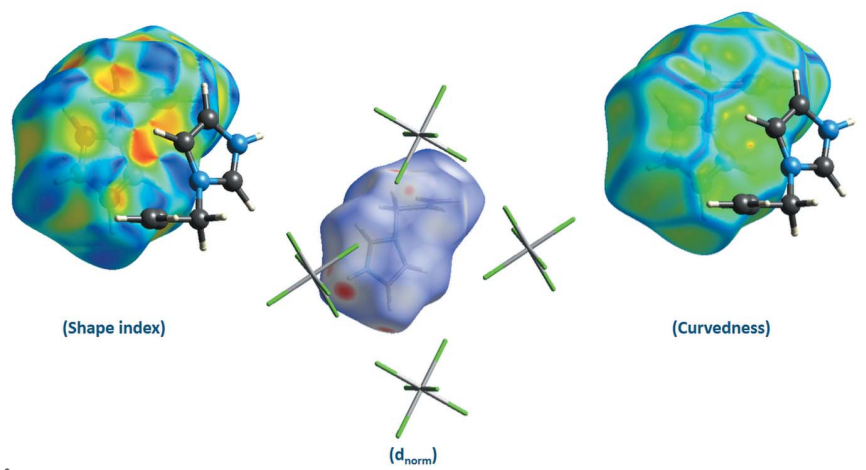

(Shape index)

Figure 4

View of the Hirshfeld surfaces for $\left(\mathrm{C}_{6} \mathrm{H}_{9} \mathrm{~N}_{2}\right)_{2}\left[\mathrm{SnCl}_{6}\right]$ mapped over shapeindex, $d_{\text {norm }}$ and curvedness.

Table 1). Additional stabilization is provided by weak $\pi-\pi$ stacking interactions between $1 H$-imidazol-3-ium rings with a centroid-to-centroid distance of 3.996 (2) § (Fig. 3).

\section{Hirshfeld surface analysis}

The Hirshfeld surface analysis (Spackman \& Jayatilaka, 2009) was performed and the associated 2D fingerprint plots (McKinnon et al., 2007) generated using Crystal Explorer 17 (Turner et al., 2017). The Hirshfeld surface was calculated using a standard (high) surface resolution with the threedimensional (3D) $d_{\text {norm }}$ surface plotted over a fixed colour scale mapped over the range -0.208 (red) to 1.180 (blue) a.u. The $d_{\text {norm }}$ mapping indicates that strong hydrogen-bonding interactions, such as $\mathrm{N}-\mathrm{H} \cdots \mathrm{Cl}$ hydrogen bonding between chlorine atoms and imidazolium groups and $\mathrm{C}-\mathrm{H} \cdots \mathrm{Cl}$ hydrogen bonding between chlorine atoms and the hydrogen atoms of the 1-(prop-2-en-1-yl) groups, appear to be the primary interactions in the structure, seen as a bright-red area in the Hirshfeld surface (Fig. 4).
A shape-index map of the title compound was calculated in the range -0.995 to 0.996 a.u. (Fig. 4). The convex blue regions on the shape-index symbolize hydrogen-donor groups and the concave red regions symbolize hydrogen-acceptor groups. $\pi-\pi$ interactions are generally indicated by adjacent red and blue triangles on the shape-index map of the Hirshfeld surface.

A curvedness map of the title compound was generated in the range -3.411 to 0.368 a.u. (Fig. 4). The large flat region of green around the rings delineated by a blue outline on the Hirshfeld surface plotted over curvedness refer to the $\pi-\pi$ stacking interactions.

The overall 2D fingerprint plot for all contacts are shown in Fig. 5, together with their relative contributions to the Hirshfeld surface. The $2 \mathrm{D}$ fingerprint plots show that the dominant intermolecular $\mathrm{H} \cdots \mathrm{Cl}(\mathrm{N} / \mathrm{C}-\mathrm{H} \cdots \mathrm{Cl})$ and $\mathrm{H} \cdots \mathrm{H}$ interactions contribute $59.8 \%$ and $25.6 \%$, respectively, to the overall crystal packing. The fingerprint plot of $\mathrm{H} \cdots \mathrm{Cl}$ contacts, which represent the largest contribution to the Hirshfeld surfaces $(59.8 \%)$, shows two large spikes highly concentrated at the edges, having almost the same $d_{\mathrm{e}}+d_{\mathrm{i}}=$ $2.7 \AA$ (Fig. 5). The H..H interactions appear as the next largest region of the fingerprint plot $(25.6 \%)$, and have a distinct pattern with a minimum value of $d_{\mathrm{e}}=d_{\mathrm{i}}=1 \AA$ (Fig. 5). Apart from these above, $\mathrm{C} \cdots \mathrm{Cl}, \mathrm{C} \cdots \mathrm{H}, \mathrm{Cl} \cdots \mathrm{Cl}, \mathrm{Cl} \cdots \mathrm{N}$, $\mathrm{N} \cdots \mathrm{H}, \mathrm{C} \cdots \mathrm{C}$, and $\mathrm{C} \cdots \mathrm{N}$ interactions were observed, which are summarized in Fig. 5.

\section{Database survey}

In the Cambridge Structural Database (Version 5.40, November 2019; Groom et al., 2016), eight structures of transition-metal coordination compounds with the 1-allylimidazole ligand are reported. The environment for the central ion in the $\left[M L_{6}\right]^{2+}$ ion is provided by the nitrogen

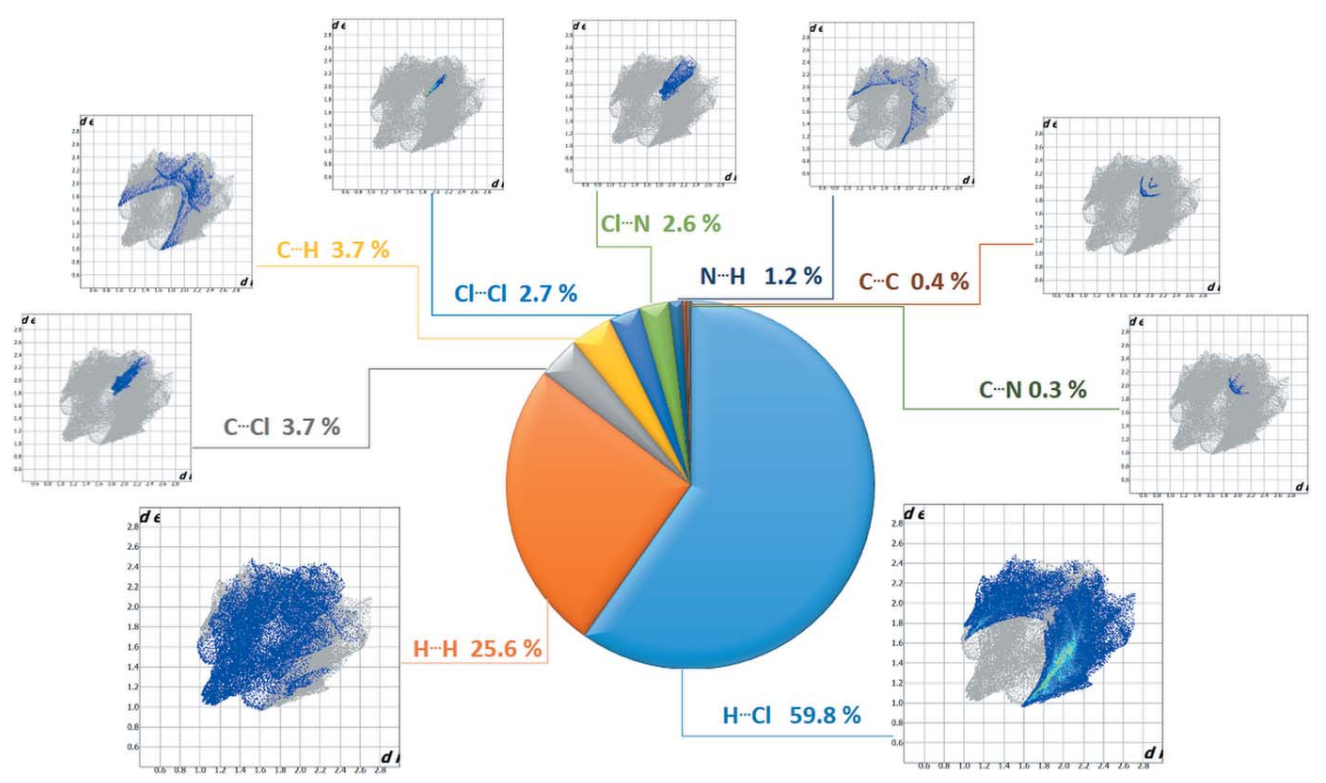

Figure 5

Two-dimensional fingerprint plots for the title compound showing the contributions of different types of interactions. 
atoms of six imidazole rings (Kurdziel \& Glowiak, 2000; Kurdziel \& Glowiak, 1998) or by other ligands with the imidazole rings (Glowiak \& Kurdziel, 2000; Curtis et al. 2008; Kurdziel \& Glowiak, 1998; Li \& Liu, 2010). However, there is no structure reported of a post-transition-metal complex with 1-allylimidazole as ligand. One bismuth complex with 1-allylimidazole $\left(\mathrm{C}_{6} \mathrm{H}_{9} \mathrm{~N}_{2}\right)_{4}\left[\mathrm{Bi}_{4} \mathrm{I}_{16}\right] \cdot 2 \mathrm{H}_{2} \mathrm{O}$ has been recently determined by Ferjani (2020), but is not yet available in the CSD. This and the title structure have the same monoclinic crystallographic $P 2_{1} / n$ symmetry. However, one has two cations in the unit cell and the other has only one. The half anionic cluster in the asymmetric unit sits on a crystallographic inversion center.

\section{UV-visible spectroscopy}

Optical absorption (OA) of the title compound was measured at ambient temperature in water. The experimental UVvisible absorption spectrum of the title compound is shown in Fig. 6. It shows one intense absorption band at $208 \mathrm{~nm}$. According to a similar compound studied previously (Maalaoui et al., 2012; Lassoued et al., 2017; Hermi et al., 2020; Mathlouthi et al., 2017), we assign this band to $\pi-\pi^{*}$ transitions within the $\left(\mathrm{C}_{6} \mathrm{H}_{9} \mathrm{~N}_{2}\right)^{+}$organic cations.

\section{Synthesis and crystallization}

The title compound was prepared by dissolving $0.34 \mathrm{~g}$ $(1 \mathrm{mmol})$ of 1-allylimidazole [1-(prop-2-en-1-yl)- $1 H$-imidazole] and $0.3 \mathrm{~g}(2 \mathrm{mmol})$ of tin(II) chloride in $10 \mathrm{ml}$ of concentrated $(37 \%)$ hydrochloric acid. The mixture was stirred with heating and then kept at room temperature. Three days later, colourless single crystals suitable for structural determination were obtained.

\section{Refinement}

Crystal data, data collection and structure refinement details are summarized in Table 2. The disordered 1-(prop-2-en-1-yl)

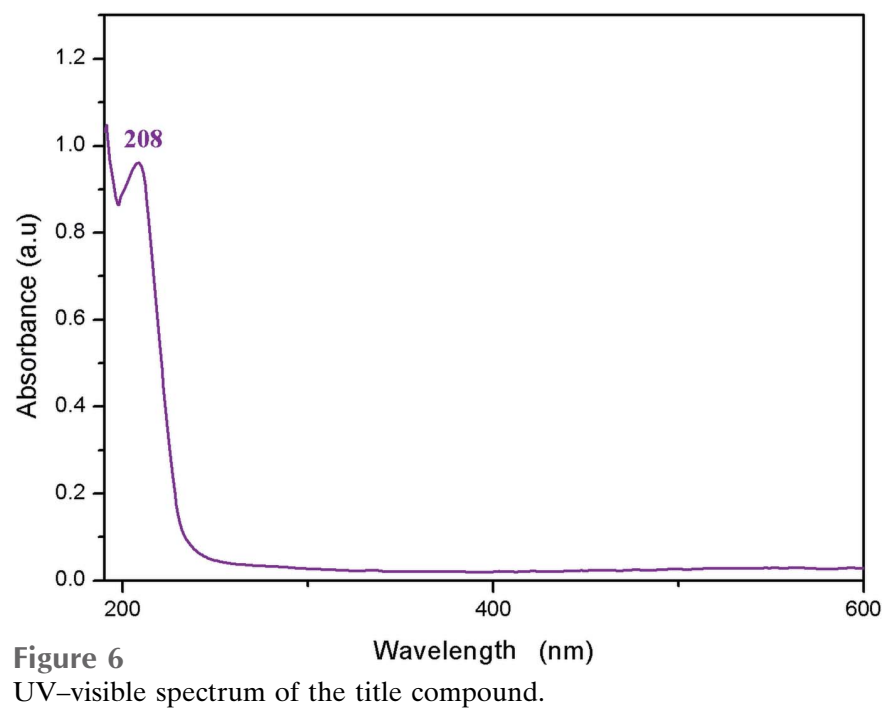

Table 2

Experimental details.

\begin{tabular}{|c|c|}
\hline \multicolumn{2}{|l|}{ Crystal data } \\
\hline Chemical formula & $\left(\mathrm{C}_{6} \mathrm{H}_{8} \mathrm{~N}_{2}\right)_{2}\left[\mathrm{SnCl}_{6}\right]$ \\
\hline$M_{\mathrm{r}}$ & 547.68 \\
\hline Crystal system, space group & Monoclinic, $P 2_{1} / n$ \\
\hline Temperature $(\mathrm{K})$ & 298 \\
\hline$a, b, c(\AA)$ & $9.3953(5), 11.7817(6), 9.8243(5)$ \\
\hline$\beta\left(^{\circ}\right)$ & $106.547(4)$ \\
\hline$V\left(\AA^{3}\right)$ & $1042.44(10)$ \\
\hline$Z$ & 2 \\
\hline Radiation type & Мо $K \alpha$ \\
\hline$\mu\left(\mathrm{mm}^{-1}\right)$ & 2.00 \\
\hline Crystal size $(\mathrm{mm})$ & $0.71 \times 0.66 \times 0.42$ \\
\hline \multicolumn{2}{|l|}{ Data collection } \\
\hline Diffractometer & Stoe IPDS2 \\
\hline Absorption correction & $\begin{array}{l}\text { Integration ( } X \text {-RED32; Stoe \& Cie, } \\
\text { 2002) }\end{array}$ \\
\hline$T_{\min }, T_{\max }$ & $0.311,0.358$ \\
\hline $\begin{array}{l}\text { No. of measured, independent and } \\
\text { observed }[I>2 \sigma(I)] \text { reflections }\end{array}$ & $12972,4294,3326$ \\
\hline$R_{\text {int }}$ & 0.035 \\
\hline$(\sin \theta / \lambda)_{\max }\left(\AA^{-1}\right)$ & 0.791 \\
\hline \multicolumn{2}{|l|}{ Refinement } \\
\hline$R\left[F^{2}>2 \sigma\left(F^{2}\right)\right], w R\left(F^{2}\right), S$ & $0.039,0.086,1.14$ \\
\hline No. of reflections & 4294 \\
\hline No. of parameters & 126 \\
\hline No. of restraints & 5 \\
\hline $\mathrm{H}$-atom treatment & H-atom parameters constrained \\
\hline$\Delta \rho_{\max }, \Delta \rho_{\min }\left(\mathrm{e} \AA^{-3}\right)$ & $0.65,-0.93$ \\
\hline
\end{tabular}

Computer programs: $X$-AREA and $X$-RED32 (Stoe \& Cie, 2002), SHELXT (Sheldrick, 2015a), SHELXL2014/7 (Sheldrick, 2015b), DIAMOND (Brandenburg, 2006) and publCIF (Westrip, 2010).

fragment in the organic cation was refined by splitting atoms $\mathrm{C} 4$ and $\mathrm{C} 5$ over two positions $(\mathrm{C} 4 A, \mathrm{C} 4 B)$ and $(\mathrm{C} 5 A, \mathrm{C} 5 B)$ with occupancy factors of 0.512 (9) and 0.488 (9). Geometrical restraints (SADI) on bond lengths were applied. $\mathrm{H}$ atoms were located in difference-Fourier maps but introduced in calculated positions and treated as riding on their parent atoms, with $\mathrm{C}-\mathrm{H}=0.93$ and $0.97 \AA, \mathrm{N}-\mathrm{H}=0.86 \AA$ with $U_{\text {iso }}(\mathrm{H})=1.2 U_{\text {eq }}(\mathrm{C}, \mathrm{N})$.

\section{Acknowledgements}

Dr Necmi Dege of the Department of Physics, Ondokuz Mayıs University (Samsun, Turkey), is thanked for performing the SCXRD and UV-visible measurements related to this work.

\section{References}

Babar, R., Munawar, M. A., Tahir, M. N. \& Arif, M. (2019). Spectrochim. Acta Part A, 217, 223-236.

BelhajSalah, S., Abdelbaky, M. S. M., García-Granda, S., Essalah, K., Ben Nasr, C. \& Mrad, M. L. (2018). Solid State Sci. 86, 77-85.

Brandenburg, K. (2006). DIAMOND. Crystal Impact GbR, Bonn, Germany.

Curtis, S. De A., Kurdziel, K., Materazzi, S. \& Vecchio, S. (2008). J. Therm. Anal. Calorim. 92, 109-114.

Ferjani, H. (2020). Crystals, 10, 397-411.

Ferjani, H., Bechaieb, R., Abd El-Fattah, W. \& Fettouhi, M. (2020). Spectrochim. Acta Part A, 161, 126-131.

Ferjani, H. \& Boughzala, H. (2018). Russ. J. Inorg. Chem. 63, 349-356.

Garci, F., Ferjani, H., Chebbi, H., Ben Jomaa, M. \& Zid, M. F. (2019). Acta Cryst. E75, 1600-1606. 
Głowiak, T. \& Kurdziel, K. (2000). J. Mol. Struct. 516, 1-5.

Groom, C. R., Bruno, I. J., Lightfoot, M. P. \& Ward, S. C. (2016). Acta Cryst. B72, 171-179.

Hajji, R., Fersi, M. A., Hajji, S., Hlel, F. \& Ben Ahmed, A. (2019). Chem. Phys. Lett. 722, 160-172.

Hajji, R., Oueslati, A., Hajlaoui, F., Bulou, A. \& Hlel, F. (2016). Phase Transit. 89, 523-542.

Hermi, S., Alotaibi, A. A., Lefebvre, F., Ben Nasr, C. \& Mrad, M. H. (2020). J. Mol. Struct. 1216, 128296.

Ishak, N. N. M., Jamsari, J., Ismail, A. Z., Tahir, M. I. M., Tiekink, E. R. T., Veerakumarasivam, A. \& Ravoof, T. B. S. A. (2019). J. Mol. Struct. 1198, 126888.

Kurdziel, K. T. \& Głowiak, T. (1998). Pol. J. Chem. 72, 2181-2181.

Kurdziel, K. T. \& Głowiak, T. (2000). Polyhedron, 19, 2183-2188.

Lassoued, M. S., Abdelbaky, M. S. M., Lassoued, A., Meroño, R. M., Ammar, S., Gadri, A., Ben Salah, A. \& García-Granda, S. (2017). J. Mol. Struct. 1141, 660-667.

Li, R.-X., Wu, Q.-Y. \& Liu, F.-Q. (2010). Acta Cryst. E66, m258.

Maalaoui, A., Olfa, B. S., Akriche, S. T., Al-Deyabd, S. S. \& Rzaigui, M. (2012). Z. Naturforsch Teil B, 67, 1178-1184.

Mathlouthi, M., Valkonen, A., Rzaigui, M. \& Smirani, W. (2017). Phase Transit. 90, 399-414.
McKinnon, J. J., Jayatilaka, D. \& Spackman, M. A. (2007). Chem. Commun. pp. 3814-3816.

Megen, M. van, Prömper, S. \& Reiss, G. J. (2013). Acta Cryst. E69, $\mathrm{m} 217$.

Parshina, L. N., Grishchenko, L. A., Smirnov, V. I., Borodina, T. N., Shakhmardanova, S. A., Tarasov, V. V., Apartsin, K. A., Kireeva, V. V. \& Trofimov, B. A. (2019). Polyhedron, 161, 126-131.

Rademeyer, M., Lemmerer, A. \& Billing, D. G. (2007). Acta Cryst. C63, m289-m292.

Sheldrick, G. M. (2015a). Acta Cryst. A71, 3-8.

Sheldrick, G. M. (2015b). Acta Cryst. C71, 3-8.

Spackman, M. A. \& Jayatilaka, D. (2009). CrystEngComm, 11, 1932.

Stoe \& Cie (2002). $X$-AREA and $X$-RED32. Stoe \& Cie GmbH, Darmstadt, Germany.

Tritt-Goc, J., Lindner, Ł., Bielejewski, M., Markiewicz, E. \& Pankiewicz, R. (2019). Carbohydr. Polym. 225, 115196.

Turner, M. J., McKinnon, J. J., Wolff, S. K., Grimwood, D. J., Spackman, P. R., Jayatilaka, D. \& Spackman, M. A. (2017). Crystal Explorer17. The University of Western Australia.

Westrip, S. P. (2010). J. Appl. Cryst. 43, 920-925.

Zhou, B. \& Liu, H. (2012). Acta Cryst. E68, m782. 


\section{supporting information}

Acta Cryst. (2020). E76, 1624-1628 [https://doi.org/10.1107/S2056989020012177]

\section{Crystal structure, optical property and Hirshfeld surface analysis of bis-}

\section{[1-(prop-2-en-1-yl)-1H-imidazol-3-ium] hexachloridostannate(IV)}

\section{Hela Ferjani}

Computing details

Data collection: $X$-AREA (Stoe \& Cie, 2002); cell refinement: $X$-AREA (Stoe \& Cie, 2002); data reduction: $X$-RED32

(Stoe \& Cie, 2002); program(s) used to solve structure: SHELXT (Sheldrick, 2015a); program(s) used to refine structure: SHELXL2014/7 (Sheldrick, 2015b); molecular graphics: DIAMOND (Brandenburg, 2006); software used to prepare material for publication: publCIF (Westrip, 2010).

Bis[1-(prop-2-en-1-yl)-1H-imidazol-3-ium] hexachloridostannate(IV)

\section{Crystal data}

$\left(\mathrm{C}_{6} \mathrm{H}_{8} \mathrm{~N}_{2}\right)_{2}\left[\mathrm{SnCl}_{6}\right]$

$M_{r}=547.68$

Monoclinic, $P 2_{1} / n$

$a=9.3953(5) \AA$

$b=11.7817(6) \AA$

$c=9.8243(5) \AA$

$\beta=106.547(4)^{\circ}$

$V=1042.44(10) \AA^{3}$

$Z=2$

\section{Data collection}

Stoe IPDS2 diffractometer

Radiation source: sealed X-ray tube, 12 x 0.4 $\mathrm{mm}$ long-fine focus

Detector resolution: 6.67 pixels $\mathrm{mm}^{-1}$ rotation method scans

Absorption correction: integration

(X-RED32; Stoe \& Cie, 2002)

$T_{\min }=0.311, T_{\max }=0.358$

Refinement

Refinement on $F^{2}$

Least-squares matrix: full

$R\left[F^{2}>2 \sigma\left(F^{2}\right)\right]=0.039$

$w R\left(F^{2}\right)=0.086$

$S=1.14$

4294 reflections

126 parameters

5 restraints
$F(000)=536$

$D_{\mathrm{x}}=1.745 \mathrm{Mg} \mathrm{m}^{-3}$

Mo $K \alpha$ radiation, $\lambda=0.71073 \AA$

Cell parameters from 19596 reflections

$\theta=2.3-34.4^{\circ}$

$\mu=2.00 \mathrm{~mm}^{-1}$

$T=298 \mathrm{~K}$

Prism, colorless

$0.71 \times 0.66 \times 0.42 \mathrm{~mm}$

12972 measured reflections

4294 independent reflections

3326 reflections with $I>2 \sigma(I)$

$R_{\text {int }}=0.035$

$\theta_{\max }=34.2^{\circ}, \theta_{\min }=2.7^{\circ}$

$h=-11 \rightarrow 14$

$k=-17 \rightarrow 18$

$l=-15 \rightarrow 15$

Hydrogen site location: inferred from neighbouring sites

$\mathrm{H}$-atom parameters constrained

$w=1 /\left[\sigma^{2}\left(F_{\mathrm{o}}^{2}\right)+(0.0288 P)^{2}+0.5447 P\right]$

where $P=\left(F_{\mathrm{o}}{ }^{2}+2 F_{\mathrm{c}}{ }^{2}\right) / 3$

$(\Delta / \sigma)_{\max }<0.001$

$\Delta \rho_{\max }=0.65$ e $\AA^{-3}$

$\Delta \rho_{\min }=-0.93$ e $\AA^{-3}$ 
Extinction correction: SHELXL-2014/7

(Sheldrick 2014,

$\mathrm{Fc}^{*}=\mathrm{kFc}\left[1+0.001 \mathrm{xFc}^{2} \lambda^{3} / \sin (2 \theta)\right]^{-1 / 4}$

Extinction coefficient: $0.0276(10)$

\section{Special details}

Geometry. All esds (except the esd in the dihedral angle between two 1.s. planes) are estimated using the full covariance matrix. The cell esds are taken into account individually in the estimation of esds in distances, angles and torsion angles; correlations between esds in cell parameters are only used when they are defined by crystal symmetry. An approximate (isotropic) treatment of cell esds is used for estimating esds involving l.s. planes.

Fractional atomic coordinates and isotropic or equivalent isotropic displacement parameters $\left(\AA^{2}\right)$

\begin{tabular}{llllll}
\hline & $x$ & $y$ & $z$ & $U_{\text {iso }} * / U_{\text {eq }}$ & Occ. $(<1)$ \\
\hline Sn1 & 1.0000 & 0.5000 & 0.5000 & $0.03804(7)$ & \\
C12 & $0.87600(8)$ & $0.32725(6)$ & $0.39099(8)$ & $0.05747(17)$ & \\
Cl3 & $1.12514(8)$ & $0.39362(6)$ & $0.70986(7)$ & $0.05588(17)$ & \\
C11 & $0.79125(8)$ & $0.53542(7)$ & $0.59310(8)$ & $0.06053(18)$ & \\
N2 & $0.3752(3)$ & $0.5613(2)$ & $0.1386(4)$ & $0.0803(9)$ & \\
N1 & $0.5438(4)$ & $0.4614(4)$ & $0.2679(4)$ & $0.0884(10)$ & \\
H1A & 0.6204 & 0.4413 & 0.3351 & $0.106^{*}$ & \\
C2 & $0.4639(4)$ & $0.3926(3)$ & $0.1674(5)$ & $0.0778(10)$ & \\
H2 & 0.4800 & 0.3156 & 0.1572 & $0.093^{*}$ & \\
C3 & $0.3583(4)$ & $0.4548(3)$ & $0.0861(4)$ & $0.0718(9)$ & \\
H3 & 0.2849 & 0.4301 & 0.0065 & $0.086^{*}$ & \\
C1 & $0.4905(5)$ & $0.5607(4)$ & $0.2501(5)$ & $0.0895(13)$ & \\
H1 & 0.5276 & 0.6231 & 0.3073 & $0.107^{*}$ & \\
C6 & $0.0239(5)$ & $0.6629(5)$ & $-0.0277(6)$ & $0.1064(17)$ & \\
H6A & 0.0288 & 0.6570 & -0.1207 & $0.128^{*}$ & $0.512(9)$ \\
H6B & -0.0676 & 0.6696 & -0.0097 & $0.128^{*}$ & $0.512(9)$ \\
C5A & $0.1409(7)$ & $0.6617(7)$ & $0.0733(9)$ & $0.0645(19)$ & $0.488(9)$ \\
H5A & 0.1396 & 0.6674 & 0.1674 & $0.077^{*}$ & $0.488(9)$ \\
C4A & $0.2828(8)$ & $0.6505(7)$ & $0.0334(13)$ & $0.085(3)$ & $0.512(9)$ \\
H4A1 & 0.3346 & 0.7226 & 0.0435 & $0.102^{*}$ & $0.512(9)$ \\
H4A2 & 0.2635 & 0.6247 & -0.0639 & $0.102^{*}$ & $0.512(9)$ \\
C4B & $0.2765(9)$ & $0.6630(6)$ & $0.1398(10)$ & $0.073(2)$ & $0.488(9)$ \\
H4B1 & 0.2290 & 0.6558 & 0.2150 & $0.087^{*}$ & $0.488(9)$ \\
H4B2 & 0.3340 & 0.7326 & 0.1543 & $0.087^{*}$ & $0.488(9)$ \\
C5B & $0.1662(11)$ & $0.6638(8)$ & $0.0018(11)$ & $0.086(3)$ & $0.512(9)$ \\
H5B & 0.2039 & 0.6651 & -0.0761 & $0.103^{*}$ & $0.512(9)$ \\
& & & & & \\
\hline
\end{tabular}

Atomic displacement parameters $\left(\AA^{2}\right)$

\begin{tabular}{lllllll}
\hline & $U^{11}$ & $U^{22}$ & $U^{33}$ & $U^{12}$ & $U^{13}$ & $U^{23}$ \\
\hline Sn1 & $0.04007(11)$ & $0.03416(10)$ & $0.03755(11)$ & $0.00060(9)$ & $0.00730(7)$ & $0.00264(8)$ \\
C12 & $0.0611(4)$ & $0.0399(3)$ & $0.0626(4)$ & $-0.0058(3)$ & $0.0033(3)$ & $-0.0064(3)$ \\
C13 & $0.0597(4)$ & $0.0527(3)$ & $0.0467(3)$ & $0.0001(3)$ & $0.0015(3)$ & $0.0136(3)$ \\
C11 & $0.0553(4)$ & $0.0704(4)$ & $0.0611(4)$ & $0.0057(3)$ & $0.0250(3)$ & $-0.0003(3)$ \\
N2 & $0.0484(14)$ & $0.0470(14)$ & $0.140(3)$ & $-0.0018(11)$ & $0.0178(16)$ & $0.0098(16)$
\end{tabular}


supporting information

\begin{tabular}{lllllll}
$\mathrm{N} 1$ & $0.0568(17)$ & $0.107(3)$ & $0.090(2)$ & $-0.0018(19)$ & $0.0023(15)$ & $0.016(2)$ \\
$\mathrm{C} 2$ & $0.071(2)$ & $0.0554(19)$ & $0.111(3)$ & $0.0096(17)$ & $0.033(2)$ & $0.005(2)$ \\
$\mathrm{C} 3$ & $0.069(2)$ & $0.067(2)$ & $0.071(2)$ & $-0.0158(17)$ & $0.0064(16)$ & $-0.0003(17)$ \\
$\mathrm{C} 1$ & $0.066(2)$ & $0.086(3)$ & $0.117(3)$ & $-0.024(2)$ & $0.027(2)$ & $-0.038(3)$ \\
$\mathrm{C} 6$ & $0.078(3)$ & $0.124(4)$ & $0.107(3)$ & $-0.015(3)$ & $0.009(2)$ & $0.037(3)$ \\
$\mathrm{C} 5 \mathrm{~A}$ & $0.064(4)$ & $0.076(4)$ & $0.059(4)$ & $0.012(3)$ & $0.028(3)$ & $0.014(3)$ \\
$\mathrm{C} 4 \mathrm{~A}$ & $0.068(4)$ & $0.068(4)$ & $0.127(9)$ & $0.020(3)$ & $0.042(5)$ & $0.045(5)$ \\
$\mathrm{C} 4 \mathrm{~B}$ & $0.075(5)$ & $0.052(4)$ & $0.081(6)$ & $0.000(3)$ & $0.005(4)$ & $0.002(3)$ \\
$\mathrm{C} 5 \mathrm{~B}$ & $0.099(7)$ & $0.078(5)$ & $0.072(5)$ & $0.010(5)$ & $0.010(5)$ & $0.023(4)$ \\
\hline
\end{tabular}

Geometric parameters $\left(A,{ }^{\circ}\right)$

\begin{tabular}{|c|c|c|c|}
\hline $\mathrm{Sn} 1-\mathrm{Cl} 3$ & $2.4131(6)$ & $\mathrm{C} 3-\mathrm{H} 3$ & 0.9300 \\
\hline $\mathrm{Sn} 1-\mathrm{Cl}^{\mathrm{i}}$ & $2.4131(6)$ & $\mathrm{C} 1-\mathrm{H} 1$ & 0.9300 \\
\hline $\mathrm{Sn} 1-\mathrm{Cl1}^{\mathrm{i}}$ & $2.4247(7)$ & $\mathrm{C} 6-\mathrm{C} 5 \mathrm{~A}$ & $1.253(8)$ \\
\hline $\mathrm{Sn} 1-\mathrm{Cl1}$ & $2.4247(7)$ & $\mathrm{C} 6-\mathrm{C} 5 \mathrm{~B}$ & $1.285(10)$ \\
\hline $\mathrm{Sn} 1-\mathrm{Cl} 2$ & $2.4363(6)$ & C6-H6A & 0.9300 \\
\hline $\mathrm{Sn} 1-\mathrm{Cl} 2^{\mathrm{i}}$ & $2.4363(6)$ & $\mathrm{C} 6-\mathrm{H} 6 \mathrm{~B}$ & 0.9300 \\
\hline $\mathrm{N} 2-\mathrm{C} 1$ & $1.303(5)$ & $\mathrm{C} 5 \mathrm{~A}-\mathrm{C} 4 \mathrm{~A}$ & $1.499(9)$ \\
\hline $\mathrm{N} 2-\mathrm{C} 3$ & $1.349(5)$ & $\mathrm{C} 5 \mathrm{~A}-\mathrm{H} 5 \mathrm{~A}$ & 0.9300 \\
\hline $\mathrm{N} 2-\mathrm{C} 4 \mathrm{~B}$ & $1.517(8)$ & $\mathrm{C} 4 \mathrm{~A}-\mathrm{H} 4 \mathrm{~A} 1$ & 0.9700 \\
\hline $\mathrm{N} 2-\mathrm{C} 4 \mathrm{~A}$ & $1.554(8)$ & $\mathrm{C} 4 \mathrm{~A}-\mathrm{H} 4 \mathrm{~A} 2$ & 0.9700 \\
\hline $\mathrm{N} 1-\mathrm{C} 1$ & $1.265(6)$ & $\mathrm{C} 4 \mathrm{~B}-\mathrm{C} 5 \mathrm{~B}$ & $1.453(9)$ \\
\hline $\mathrm{N} 1-\mathrm{C} 2$ & $1.331(5)$ & $\mathrm{C} 4 \mathrm{~B}-\mathrm{H} 4 \mathrm{~B} 1$ & 0.9700 \\
\hline $\mathrm{N} 1-\mathrm{H} 1 \mathrm{~A}$ & 0.8600 & $\mathrm{C} 4 \mathrm{~B}-\mathrm{H} 4 \mathrm{~B} 2$ & 0.9700 \\
\hline $\mathrm{C} 2-\mathrm{C} 3$ & $1.307(5)$ & $\mathrm{C} 5 \mathrm{~B}-\mathrm{H} 5 \mathrm{~B}$ & 0.9300 \\
\hline $\mathrm{C} 2-\mathrm{H} 2$ & 0.9300 & & \\
\hline $\mathrm{Cl} 3-\mathrm{Sn} 1-\mathrm{Cl}^{\mathrm{i}}$ & 180.0 & $\mathrm{C} 2-\mathrm{C} 3-\mathrm{H} 3$ & 126.2 \\
\hline $\mathrm{Cl} 3-\mathrm{Sn} 1-\mathrm{Cl1}{ }^{\mathrm{i}}$ & $89.04(3)$ & $\mathrm{N} 2-\mathrm{C} 3-\mathrm{H} 3$ & 126.2 \\
\hline $\mathrm{Cl} 3^{\mathrm{i}}-\mathrm{Sn} 1-\mathrm{Cl} 1^{\mathrm{i}}$ & $90.96(3)$ & $\mathrm{N} 1-\mathrm{C} 1-\mathrm{N} 2$ & $108.8(4)$ \\
\hline $\mathrm{Cl} 3-\mathrm{Sn} 1-\mathrm{Cl} 1$ & $90.96(3)$ & $\mathrm{N} 1-\mathrm{C} 1-\mathrm{H} 1$ & 125.6 \\
\hline $\mathrm{Cl} 3^{\mathrm{i}}-\mathrm{Sn} 1-\mathrm{Cl} 1$ & $89.04(3)$ & $\mathrm{N} 2-\mathrm{C} 1-\mathrm{H} 1$ & 125.6 \\
\hline $\mathrm{Cl1}{ }^{\mathrm{i}}-\mathrm{Sn} 1-\mathrm{Cl} 1$ & 180.0 & $\mathrm{C} 5 \mathrm{~A}-\mathrm{C} 6-\mathrm{H} 6 \mathrm{~A}$ & 120.0 \\
\hline $\mathrm{Cl} 3-\mathrm{Sn} 1-\mathrm{Cl} 2$ & $89.85(2)$ & $\mathrm{C} 5 \mathrm{~A}-\mathrm{C} 6-\mathrm{H} 6 \mathrm{~B}$ & 120.0 \\
\hline $\mathrm{Cl} 3^{\mathrm{i}}-\mathrm{Sn} 1-\mathrm{Cl} 2$ & $90.15(2)$ & $\mathrm{H} 6 \mathrm{~A}-\mathrm{C} 6-\mathrm{H} 6 \mathrm{~B}$ & 120.0 \\
\hline $\mathrm{C} 11^{\mathrm{i}}-\mathrm{Sn} 1-\mathrm{Cl} 2$ & $91.56(3)$ & $\mathrm{C} 6-\mathrm{C} 5 \mathrm{~A}-\mathrm{C} 4 \mathrm{~A}$ & $116.0(7)$ \\
\hline $\mathrm{Cl} 1-\mathrm{Sn} 1-\mathrm{Cl} 2$ & $88.44(3)$ & $\mathrm{C} 6-\mathrm{C} 5 \mathrm{~A}-\mathrm{H} 5 \mathrm{~A}$ & 122.0 \\
\hline $\mathrm{Cl} 3-\mathrm{Sn} 1-\mathrm{Cl} 2^{\mathrm{i}}$ & $90.15(2)$ & $\mathrm{C} 4 \mathrm{~A}-\mathrm{C} 5 \mathrm{~A}-\mathrm{H} 5 \mathrm{~A}$ & 122.0 \\
\hline $\mathrm{Cl} 3^{\mathrm{i}}-\mathrm{Sn} 1-\mathrm{Cl} 2^{\mathrm{i}}$ & $89.85(2)$ & $\mathrm{C} 5 \mathrm{~A}-\mathrm{C} 4 \mathrm{~A}-\mathrm{N} 2$ & $104.8(6)$ \\
\hline $\mathrm{C} 11^{\mathrm{i}}-\mathrm{Sn} 1-\mathrm{Cl} 2^{\mathrm{i}}$ & $88.44(3)$ & $\mathrm{C} 5 \mathrm{~A}-\mathrm{C} 4 \mathrm{~A}-\mathrm{H} 4 \mathrm{~A} 1$ & 110.8 \\
\hline $\mathrm{Cl} 1-\mathrm{Sn} 1-\mathrm{Cl} 2^{\mathrm{i}}$ & $91.56(3)$ & $\mathrm{N} 2-\mathrm{C} 4 \mathrm{~A}-\mathrm{H} 4 \mathrm{~A} 1$ & 110.8 \\
\hline $\mathrm{Cl} 2-\mathrm{Sn} 1-\mathrm{Cl} 2^{\mathrm{i}}$ & 180.0 & $\mathrm{C} 5 \mathrm{~A}-\mathrm{C} 4 \mathrm{~A}-\mathrm{H} 4 \mathrm{~A} 2$ & 110.8 \\
\hline $\mathrm{C} 1-\mathrm{N} 2-\mathrm{C} 3$ & $107.2(3)$ & $\mathrm{N} 2-\mathrm{C} 4 \mathrm{~A}-\mathrm{H} 4 \mathrm{~A} 2$ & 110.8 \\
\hline $\mathrm{C} 1-\mathrm{N} 2-\mathrm{C} 4 \mathrm{~B}$ & $111.2(4)$ & $\mathrm{H} 4 \mathrm{~A} 1-\mathrm{C} 4 \mathrm{~A}-\mathrm{H} 4 \mathrm{~A} 2$ & 108.9 \\
\hline $\mathrm{C} 3-\mathrm{N} 2-\mathrm{C} 4 \mathrm{~B}$ & $137.0(4)$ & $\mathrm{C} 5 \mathrm{~B}-\mathrm{C} 4 \mathrm{~B}-\mathrm{N} 2$ & $105.8(7)$ \\
\hline $\mathrm{C} 1-\mathrm{N} 2-\mathrm{C} 4 \mathrm{~A}$ & $137.4(5)$ & $\mathrm{C} 5 \mathrm{~B}-\mathrm{C} 4 \mathrm{~B}-\mathrm{H} 4 \mathrm{~B} 1$ & 110.6 \\
\hline $\mathrm{C} 3-\mathrm{N} 2-\mathrm{C} 4 \mathrm{~A}$ & $113.1(6)$ & $\mathrm{N} 2-\mathrm{C} 4 \mathrm{~B}-\mathrm{H} 4 \mathrm{~B} 1$ & 110.6 \\
\hline
\end{tabular}




$\begin{array}{llll}\mathrm{C} 1-\mathrm{N} 1-\mathrm{C} 2 & 110.1(3) & \mathrm{C} 5 \mathrm{~B}-\mathrm{C} 4 \mathrm{~B}-\mathrm{H} 4 \mathrm{~B} 2 & 110.6 \\ \mathrm{C} 1-\mathrm{N} 1-\mathrm{H} 1 \mathrm{~A} & 124.9 & \mathrm{~N} 2-\mathrm{C} 4 \mathrm{~B}-\mathrm{H} 4 \mathrm{~B} 2 & 110.6 \\ \mathrm{C} 2-\mathrm{N} 1-\mathrm{H} 1 \mathrm{~A} & 124.9 & \mathrm{H} 4 \mathrm{~B} 1-\mathrm{C} 4 \mathrm{~B}-\mathrm{H} 4 \mathrm{~B} 2 & 108.7 \\ \mathrm{C} 3-\mathrm{C} 2-\mathrm{N} 1 & 106.3(3) & \mathrm{C} 6-\mathrm{C} 5 \mathrm{~B}-\mathrm{C} 4 \mathrm{~B} & 129.0(10) \\ \mathrm{C} 3-\mathrm{C} 2-\mathrm{H} 2 & 126.8 & \mathrm{C} 6-\mathrm{C} 5 \mathrm{~B}-\mathrm{H} 5 \mathrm{~B} & 115.5 \\ \mathrm{~N} 1-\mathrm{C} 2-\mathrm{H} 2 & 126.8 & \mathrm{C} 4 \mathrm{~B}-\mathrm{C} 5 \mathrm{~B}-\mathrm{H} 5 \mathrm{~B} & 115.5 \\ \mathrm{C} 2-\mathrm{C} 3-\mathrm{N} 2 & 107.6(3) & & \\ & & & -160.5(6) \\ \mathrm{C} 1-\mathrm{N} 1-\mathrm{C} 2-\mathrm{C} 3 & 0.2(5) & \mathrm{C} 4 \mathrm{~A}-\mathrm{N} 2-\mathrm{C} 1-\mathrm{N} 1 & -135.5(8) \\ \mathrm{N} 1-\mathrm{C} 2-\mathrm{C} 3-\mathrm{N} 2 & -0.1(5) & \mathrm{C} 1-\mathrm{N} 2-\mathrm{C} 4 \mathrm{~A}-\mathrm{N}-\mathrm{N} 2 & -106.0(7) \\ \mathrm{C} 1-\mathrm{N} 2-\mathrm{C} 3-\mathrm{C} 2 & 0.0(5) & \mathrm{C} 3-\mathrm{N} 2-\mathrm{C} 4 \mathrm{~A}-\mathrm{C} 5 \mathrm{~A} & 94.2(8) \\ \mathrm{C} 4 \mathrm{~B}-\mathrm{N} 2-\mathrm{C} 3-\mathrm{C} 2 & -152.5(7) & \mathrm{C} 1-\mathrm{N} 2-\mathrm{C} 4 \mathrm{~B}-\mathrm{C} 5 \mathrm{~B} & 171.4(6) \\ \mathrm{C} 4 \mathrm{~A}-\mathrm{N} 2-\mathrm{C} 3-\mathrm{C} 2 & 165.9(4) & \mathrm{C} 3-\mathrm{N} 2-\mathrm{C} 4 \mathrm{~B}-\mathrm{C} 5 \mathrm{~B} & -36.7(11) \\ \mathrm{C} 2-\mathrm{N} 1-\mathrm{C} 1-\mathrm{N} 2 & -0.1(5) & \mathrm{N} 2-\mathrm{C} 4 \mathrm{~B}-\mathrm{C} 5 \mathrm{~B}-\mathrm{C} 6 & 124.1(9) \\ \mathrm{C} 3-\mathrm{N} 2-\mathrm{C} 1-\mathrm{N} 1 & 0.1(5) & & \\ \mathrm{C} 4 \mathrm{~B}-\mathrm{N} 2-\mathrm{C} 1-\mathrm{N} 1 & 160.4(5) & & \end{array}$

Symmetry code: (i) $-x+2,-y+1,-z+1$.

Hydrogen-bond geometry $\left(\AA,{ }^{\circ}\right)$

\begin{tabular}{lllll}
\hline$D-\mathrm{H} \cdots A$ & $D-\mathrm{H}$ & $\mathrm{H} \cdots A$ & $D \cdots A$ & $D-\mathrm{H} \cdots A$ \\
\hline $\mathrm{N} 1-\mathrm{H} 1 A \cdots \mathrm{Cl} 2$ & 0.86 & 2.67 & $3.399(4)$ & 144 \\
$\mathrm{~N} 1-\mathrm{H} 1 A \cdots \mathrm{Cl1}$ & 0.86 & 2.81 & $3.485(4)$ & 136 \\
$\mathrm{C} 2-\mathrm{H} 2 \cdots \mathrm{Cl} 3^{\mathrm{ii}}$ & 0.93 & 2.79 & $3.672(4)$ & 158 \\
$\mathrm{C} 3-\mathrm{H} 3 \cdots \mathrm{Cl} 3{ }^{\mathrm{iii}}$ & 0.93 & 2.90 & $3.790(4)$ & 160 \\
$\mathrm{C} 4 A-\mathrm{H} 4 A 1 \cdots \mathrm{Cl}{ }^{\mathrm{iv}}$ & 0.97 & 2.89 & $3.716(9)$ & 144 \\
$\mathrm{C} 4 A-\mathrm{H} 4 A 1 \cdots \mathrm{Cl} 1^{\mathrm{v}}$ & 0.97 & 2.94 & $3.743(10)$ & 141 \\
$\mathrm{C} 4 B-\mathrm{H} 4 B 1 \cdots \mathrm{Cl} 1^{\mathrm{vi}}$ & 0.97 & 2.98 & $3.702(10)$ & 133 \\
$\mathrm{C} 4 B-\mathrm{H} 4 B 2 \cdots \mathrm{Cl} 1^{\mathrm{v}}$ & 0.97 & 2.80 & $3.590(7)$ & 139 \\
$\mathrm{C} 5 B-\mathrm{H} 5 B \cdots \mathrm{Cl} 2^{\text {vii }}$ & 0.93 & 2.97 & $3.766(11)$ & 145 \\
\hline
\end{tabular}

Symmetry codes: (ii) $x-1 / 2,-y+1 / 2, z-1 / 2$; (iii) $x-1, y, z-1$; (iv) $-x+3 / 2, y+1 / 2,-z+1 / 2$; (v) $x-1 / 2,-y+3 / 2, z-1 / 2$; (vi) $-x+1,-y+1,-z+1$; (vii) $-x+1$, $-y+1,-z$. 